\title{
Lattice gas models of coherent strained epitaxy
}

\author{
V. I. Tokar ${ }^{1,2}$ and H. Dreyssé ${ }^{1}$ \\ 1 IPCMS-GEMM, UMR 7504 CNRS, 23, \\ rue du Loess, F-6r037 Strasbourg Cedex, France \\ ${ }^{2}$ Institute of Magnetism, National Academy of Sciences, \\ 36-b Vernadsky str., 03142 Kiev-142, Ukraine
}

(Dated: November 20, 2018)

\begin{abstract}
The harmonic Frenkel-Kontorova model is used to illustrate with an exactly solvable example a general technique of mapping a coherently strained epitaxial system with continuous atomic displacements onto a lattice gas model (LGM) with only discrete variables. The misfit strain of the original model is transformed into cluster interatomic interactions of the LGM. The clusters are contiguous atomic chains of all lengths but the interaction strength for long chains is exponentially small. This makes possible the application of efficient Monte Carlo techniques developed for discrete variables both in kinetic and equilibrium simulations. The formalism developed can be applied to $1 \mathrm{D}$ as well as to $2 \mathrm{D}$ systems. As an illustrative example we consider the problem of self-organization of 1D size calibrated clusters on the steps of the vicinal surfaces.
\end{abstract}

PACS numbers: 05.65.+b, 68.66.La, 81.16.Dn 


\section{INTRODUCTION}

The phenomena of self-assembly and self-organization of coherent (i. e., dislocation-free) size calibrated nano- and atomic-scale structures observed during the heteroepitaxial growth in some systems 1 are considered to be promising tools for fabrication of microelectronic devices].

A major factor influencing the phenomenon of self-assembly is the lattice size misfit (LSM) between the substrate and the growing overlayer commonly encountered in heteroepitaxial systems ${ }^{-}$. The LSM-induced strain is believed to be the driving force behind the above phenomenal. basis of any theory of strained epitaxy. Because strained systems exhibit complicated kinetics and morphologies, analytic approach is difficult, so a major technique in theoretical studies of strained epitaxy is the kinetic Monte Carlo simulation. The application of this technique, however, is severely hampered by the necessity to simulate the continuous atomic displacements. Because of this, atomistic models in such simulations are currently restricted to rather small systems consisting of only a few thousand atoms served 3D quantum dots sometimes consist of several tens of thousand atoms eachl.

Our research is based on the observation that as long as we are interested only in the coherent structures, there is a possibility to map the system onto a purely lattice model because in the absence of dislocations the relaxed atom can only either deviate from its symmetric position inside the same cell or to be displaced to another cell but there always exists a lattice site of a regular lattice to which this atom can be ascribed. So our first goal is to develop a formalism which would allow to map a coherent heteroepitaxial system with the continuous variables onto a lattice gas model with only discrete variables.

One of important goals of the heteroepitaxial studies is the development of techniques of growth of 1D quantum wires (QW) which can be used, e. g., for experimental investigation of the Luttinger model of interacting 1D electronst. Furthermore, the QW may find application in microelectronics circuitryb, as well as in magnetic memory devices $\mathrm{Q}$. The latter applications would require the 1D structures of finite length. In the case of the memory devices it is also desirable that these atomic clusters (or chains) were of similar length and that they were arranged into periodic arrays in order to simplify the memory access. All these requirements can be satisfied by self-organized size calibrated structures similar to 
quantum dots of the 2D epitaxy?. A phenomenological theory explaining the mechanism of

formation of quantum dots was proposed in Ref. applied to objects in any number of dimensions. So to illustrate the techniques developed in the present paper we will study the conditions of formation of the 1D size calibrated monatomic chains.

\section{THE MODEL}

A simple approach to theoretical description of the misfit is provided by the 1D Frenkel-Kontorova model (FKM) which is frequently been used in qualitatives 10 and semiquantitative studies 11 of strained epitaxy. To illustrate the generality of our approach we first present the formalism appropriate to the 2D epitaxy but in concrete examples we will restrict ourselves to the $1 \mathrm{D}$ case.

We consider an ensemble of a fixed number $(N)$ of atoms coherently deposited on a surface with a square lattice of deposition sites. The rectangular lattice geometry was chosen because it allows for the separation of $x$ and $y$ variables (see Ref. 9 and below). Let us first consider more general model with the energy of the epilayer of the form

$$
U=\sum_{\mathbf{i}} n_{\mathbf{i}} V_{s}\left(\mathbf{R}_{\mathbf{i}}+\mathbf{u}_{\mathbf{i}}\right)+\frac{1}{2} \sum_{\mathbf{i j}} n_{\mathbf{i}} n_{\mathbf{j}} V\left(\mathbf{u}_{\mathbf{i}}+\mathbf{R}_{\mathbf{i}}-\mathbf{u}_{\mathbf{j}}-\mathbf{R}_{\mathbf{j}}\right),
$$

where $\mathbf{R}_{\mathbf{i}}=\left(a^{x} i_{x}, a^{y} i_{y}\right)$ ( $a^{\gamma}$ the lattice constants in the two directions, $i_{x}$ and $i_{y}$ integers ), $n_{\mathbf{i}}=0,1$ is the occupation number of site $\mathbf{i}, \mathbf{u}_{\mathbf{i}}$ the atomic displacement, $V_{s}$ the potential of interaction with the substrate, and $V$ the interatomic potential.

The analysis of the system considerably simplifies at low temperatures where it can be approximately reduced to a lattice gas model. This is achieved by exploiting the fact that the residence time of atoms at the lattice sites can be arbitrarily large due to the Arrhenius law obeyed by the probability of activated hopping over the potential barriers separating neighboring sites 12 . The dynamics of the variables $\mathbf{u}_{\mathbf{i}}$, on the other hand, do not have any energy barriers. So at sufficiently low temperature these variables are capable of reaching their thermal equilibrium distribution during the time intervals between the atomic hops, i. e., with the atomic configuration remaining unchanged. Averaging over $\mathbf{u}_{\mathbf{i}}$ will leave us with an effective non-equilibrium free energy function $F_{\text {eff }}$ of variables $n_{\mathbf{i}}$ only:

$$
\exp \left(-F_{\mathrm{eff}} / k_{B} T\right)=\int \prod_{\left\{n_{\mathbf{i}}=1\right\}} d \mathbf{u}_{\mathbf{i}} \exp \left(-U / k_{B} T\right)
$$


This purely lattice model can be further used in both equilibrium and kinetic studies.

For the purposes of qualitative analysis it will suffice to average out the displacement variables in Eq. (1) in the harmonic approximation, i. e., by expanding $V_{s}$ and $V_{p}$ in the above equation up to the second order in the displacement variables $\mathbf{u}_{\mathbf{i}}, 1,1,13$ which is valid for small $\left|u_{i}^{\gamma}\right| / a^{\gamma}$. This allows to perform the calculation exactly (see below). However, the integration can be extended to approximately account also for anharmonic terms. This extension, besides making the approach more accurate, can also account for some qualitative phenomenall.

To facilitate comparison with other studies based on the Frenkel-Kontorova mode [0, $10,11,14$ we write the harmonic approximation as the second order power series expansion in $\mathbf{u}_{\mathbf{i}}$ for $V_{s}$ and in $\mathbf{u}_{\mathbf{i}}-\mathbf{u}_{\mathbf{i}-\gamma}$ for the pair potential $V_{p}$ :

$$
\begin{aligned}
U \approx & V_{s} N+\frac{1}{2} \sum_{\mathbf{i} \gamma} n_{\mathbf{i}} k_{s}^{\gamma}\left(u_{\mathbf{i}}^{\gamma}\right)^{2}+\frac{1}{2} \sum_{\mathbf{i j}} n_{\mathbf{i}} n_{\mathbf{j}} V_{\mathbf{i j}} \\
& +\frac{1}{2} \sum_{\mathbf{i} \gamma} k_{p}^{\gamma}\left[\left(u_{\mathbf{i}}^{\gamma}-u_{\mathbf{i}+\gamma}^{\gamma}-f_{\gamma}\right)^{2}-f_{\gamma}^{2}\right] n_{\mathbf{i}} n_{\mathbf{i}+\gamma} \\
= & V_{s} N+\frac{1}{2} \sum_{\mathbf{i} \mathbf{j}} V_{\mathbf{i j}} n_{\mathbf{i}} n_{\mathbf{j}} \\
& +\frac{1}{2} \sum_{\mathbf{i} \mathbf{j} \gamma} k_{p}^{\gamma} D_{\mathbf{i} \mathbf{j}}^{\gamma} u_{\mathbf{i}}^{\gamma} u_{\mathbf{j}}^{\gamma}+\sum_{\mathbf{i} \gamma} k_{p}^{\gamma} f_{\gamma} u_{\mathbf{i}}^{\gamma} \nabla_{\mathbf{i}}^{\gamma} n_{\mathbf{i}} n_{\mathbf{i}+\gamma}
\end{aligned}
$$

where $V_{s}$ and $V_{\mathbf{i j}}$ are the values of the substrate and the pair potentials at the lattice sites for the atom in the symmetric position (the zero order approximation), $\gamma$ denotes $x$ or $y$ component; $f_{\gamma}=-V_{\gamma}^{\prime} / V_{\gamma \gamma}^{\prime \prime}$ (where $V$ is the pair potential) is interpreted as the misfit parameter in the direction $\gamma, k_{s}^{\gamma}$ and $k_{p}^{\gamma}$ are the second derivatives of the corresponding potentials, $D_{\mathbf{i j}}^{\gamma}$ is the dimensionless dynamical matrix defined by this equality, and $\nabla_{\mathbf{i}}^{\gamma} F_{\mathbf{i}} \equiv$ $F_{\mathbf{i}}-F_{\mathbf{i}-\gamma}$. A major simplification is achieved under the nearest neighbor (NN) approximation for the relaxation because, as is seen from the last line of Eq. (2), in this case the variables $u_{\mathbf{i}}^{x}$ and $u_{\mathbf{i}}^{y}$ separate, and the relaxations along the two directions are independent.

With approximation (2) the statistical average amounts to the Gaussian integration to give

$$
\begin{aligned}
F_{\text {eff }}= & \bar{V}_{s} N+\frac{1}{2} \sum_{\mathbf{i} \mathbf{j}} n_{\mathbf{i}} n_{\mathbf{j}} V_{\mathbf{i} \mathbf{j}}-\frac{k_{B} T}{2} \sum_{\gamma} \ln \operatorname{det} G^{\gamma} \\
& -\frac{k_{p} f^{2}}{2} \sum_{\mathbf{i} \gamma \gamma} G_{\mathbf{i} \mathbf{j}}^{\gamma} \nabla_{\mathbf{i}}^{\gamma} n_{\mathbf{i}} n_{\mathbf{i}+\gamma} \nabla_{\mathbf{j}}^{\gamma} n_{\mathbf{j}} n_{\mathbf{j}+\gamma},
\end{aligned}
$$


where $G^{\gamma}=1 / D^{\gamma}$ and in $\bar{V}_{s}$ we gathered all terms which are proportional to the total particle number, such as the normalization of the determinant coming from the in-plane Gaussian integration and a similar term from the integration along $z$-direction. We do not discuss these contributions here because in the present study we restrict ourselves to systems with a fixed number of deposited atoms $N$. In case of necessity these terms can be easily recovered. We also note the entropic contribution (the second term in the first line) which naturally appears in our formalism and which was shown to be crucial for proper description of the processes of deposition 15 as well as for the correct prediction of the shape of the atomic clusters 16 .

Because of the gradient factors, only the ends of contiguous chains of atoms contribute into the last term of Eq. (3) leaving two matrix elements of $G_{\mathbf{i j}}^{\gamma}$ in the sum over ij inside every chain: the diagonal one which we denote as $G_{l}^{(0)}(l$ the length of the chain; we omit the superscript $\gamma$ to simplify notation) and the matrix element $G_{l}^{(l-1)}$ connecting the two ends of the chain. Furthermore, in the NN approximation the matrix $D$ is block-diagonal because the atoms belonging to different chains do not couple. Therefore, the determinant factorizes and the relaxation part of the free energy which consists of the terms in Eq. (3) containing $G$ takes the form

$$
\begin{aligned}
F_{\text {relax }} & =-\sum_{\text {chains }}\left\{\frac{k_{B} T}{2} \ln \operatorname{det} \frac{G_{l}}{G_{1}}+k_{p} f^{2}\left[G_{l}^{(0)}-G_{l}^{(l-1)}\right]\right\} \\
& \equiv \sum_{\text {chains }}\left(-T S_{l}+W_{l}\right) \equiv \sum_{\text {chains }} E_{l},
\end{aligned}
$$

where the summation is over the chains consisting of two or more atoms. Besides, in the first term on the right hand side (r. h. s.) we subtracted the single atom entropy term by assuming that it is accounted for in $\bar{V}_{s}$ which we discarded to simplify notation. It is easy to show that $F_{\text {relax }}$ can be expanded into an infinite sum of multiatom interactions of the form $V_{l} n_{\mathbf{i}} n_{\mathbf{i}+\hat{\gamma}} \ldots n_{\mathbf{i}+(l-1) \hat{\gamma}}$, where $\hat{\gamma}$ is the unit vector in the direction $\hat{\gamma}$. The expansion coefficients are given by the expression

$$
V_{l}=E_{l}-2 E_{l-1}+E_{l-2}
$$

valid for all $l \geq 2$ with $E_{0}=E_{1}=0$. 


\section{THE RELAXATION FREE ENERGY}

Omitting the superscript $\gamma$ to simplify notation, the matrix $D_{l}$ for chain of length $l$ is defined according to Eq. (2) as

$$
k_{p} \sum_{i j}\left(D_{l}\right)_{i j} u_{i} u_{j}=k_{s} u_{1}^{2}+k_{p}\left(u_{1}-u_{2}\right)^{2}+k_{s} u_{2}^{2}+\ldots+k_{p}\left(u_{l-1}-u_{l}\right)^{2}+k_{s} u_{l}^{2} .
$$

Hence, the square matrix $k_{p} D_{l}$ have the following structure:

$$
k_{p} D_{l}=\left(\begin{array}{ccccccc}
k_{p}+k_{s} & -k_{p} & 0 & \ldots & 0 & 0 & 0 \\
-k_{p} & 2 k_{p}+k_{s} & -k_{p} & 0 & \ldots & 0 & 0 \\
0 & -k_{p} & 2 k_{p}+k_{s} & -k_{p} & 0 & \ldots & 0 \\
\vdots & \vdots & \ddots & \ddots & \ddots & \vdots & \vdots \\
0 & \ldots & 0 & -k_{p} & 2 k_{p}+k_{s} & -k_{p} & 0 \\
0 & 0 & \ldots & 0 & -k_{p} & 2 k_{p}+k_{s} & -k_{p} \\
0 & 0 & 0 & \ldots & 0 & -k_{p} & k_{p}+k_{s} \\
0 & & & l & & &
\end{array}\right)
$$

In Appendix $\mathrm{A}$ we have shown that the matrix elements of $G_{l}=1 / D_{l}$ entering the expression for the relaxation free energy (4) can be calculated with the use of recursion formulas for the tridiagonal matrices as [see Eqs. A10 and (A11)]

$$
\left\{\begin{array}{l}
G_{l+1}^{(0)}=1 /\left(1+\alpha-d_{l}\right) \\
G_{l+1}^{(l)}=\operatorname{det} G_{l+1}=G_{l+1}^{(0)} b_{l},
\end{array}\right.
$$

where $\alpha=k_{s} / k_{p} ; d_{l}$ and $b_{l}$ are generated by the recursion relations

$$
d_{l+1}=1 /\left(2+\alpha-d_{l}\right) \quad \text { and } \quad b_{l+1}=d_{l+1} b_{l}
$$

The recursion relations (9) at large $l$ drive $d_{l}$ and $b_{l}$ to the fixed point $d_{\star}, b_{\star}$ :

$$
\begin{aligned}
& d_{\star}=1+\frac{\alpha}{2}-\sqrt{\alpha+\frac{\alpha^{2}}{4}}<1 \\
& b_{\star}=0 .
\end{aligned}
$$

This means that at large $l G_{l}^{(0)}$ saturates to a constant value while $G_{l}^{(l)}=\operatorname{det} G_{l} \sim d_{\star}^{l}$ [see Eqs. (8)]. This in particular means that the entropic contribution into $F_{\text {relax }}$ which is proportional to $\ln \left(\operatorname{det} G_{l}\right)$ is asymptotically linear in $l$ (see Fig. 1). 
Two contributions into $F_{\text {relax }}$ calculated with these formulas are shown in Fig. [. The entropic contribution is negative because in our canonical ensemble formalism we discarded the entropy corresponding to $N$ isolated atoms which is reflected in the denominator $G_{1}$ in the expression (14) for the free energy. This means, in particular, that when $k_{p} \rightarrow 0$ the atomic displacements within the cell became mutually independent (the on-site pair interactions do not depend on the atomic positions inside the cell), so $G_{l} \rightarrow G_{1} \hat{I}$, where $\hat{I}$ is the unit matrix, and the entropy term tends to zero [see Eq. (四) and Fig. 国. When $k_{p}$ is not equal to zero, the atomic displacements became restricted by its neighbors, so their entropy diminishes. For large chains the interior atoms are practically in translationally invariant environment, so for long chains the entropy loss is proportional to $l$. The above restrictions on the atomic displacements are the more stringent the larger $k_{p}$. This is reflected in the slope of $S_{l}$ which is larger for smaller $\alpha=k_{s} / k_{p}$ (see Fig. (1).

Because the entropic contribution in Eq. (四) is multiplied by $T$, at low temperature it can be neglected. The remaining term $W_{l}$ is negative and saturates to a finite value at large $l$ (see Fig. [1b). From Eq. (2) it follows that the NN interatomic interaction $V_{\mathrm{NN}}=V_{\mathrm{NN}}^{\text {ch }}+k_{p} f^{2}$, where the first term $\left(V_{\mathrm{NN}}^{\text {ch }}\right)$ is some "chemical" interaction and the second term is the unrelaxed repulsion due to LSM. Thus, the relaxation energy in Eq. (四) is the difference between the relaxed elastic energy of the chain $E_{r e l}^{e l}(l)$ and the unrelaxed one $E_{0}^{e l}(l)=(l-1) k_{p} f^{2}$ with both energies being positive, as it should be for elastic energies. Thus,

$$
W_{l}=E_{r e l}^{e l}(l)-E_{0}^{e l}(l)
$$

and is negative because relaxation lowers the energy. Now, if $V_{\mathrm{NN}}^{\mathrm{ch}}$ is negative but such that $V_{\mathrm{NN}}$ is not too large, then the reduced chain energy $\left[W_{l}+V_{\mathrm{NN}}(l-1)\right] / l$ will have a minimum corresponding to an equilibrium chain length. In two dimensions these chains will unify in square platelets. Thus, our model contains the mechanism for the size calibration of atomic clusters which was phenomenologically described in Ref. 目. In 2D this conclusion as well as the formula of Refl目 were verified with the use of the Monte Carlo simulation 17 .

Because the entropic contribution $S_{l}$ (see Fig. [a) is practically linear in $l$ according to Eq. (5) which has the form of the discrete second derivative it essentially contributes only into the pair interaction $V_{2}$ (the multiatom contributions were found to be $\sim 10 \%$ ). Thus, the relaxation entropy formally amounts to effective NN interatomic repulsion which grows linearly with temperature. The entropic forces of this kind were earlier discovered in alloys 


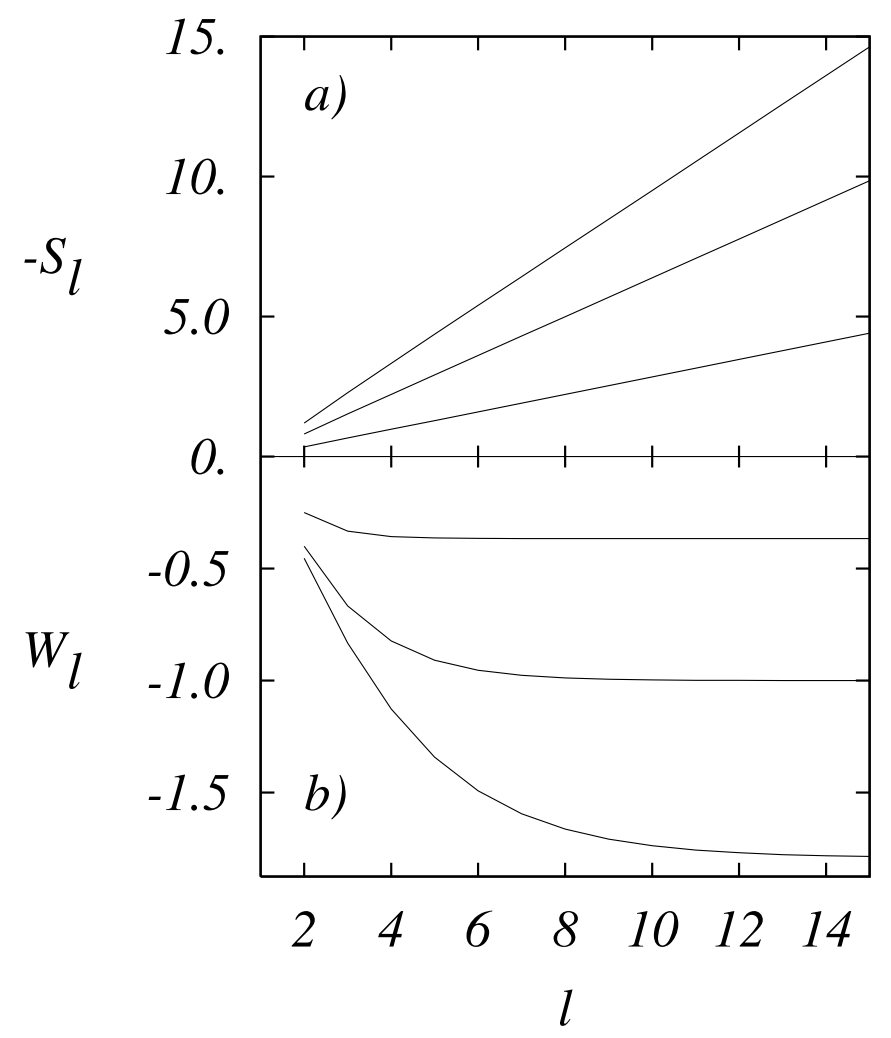

FIG. 1: Relaxation entropy in units of $k_{B}$ (a) and relaxation energy in units of $k_{p} f^{2}$ (b) for chains of length $l$. At both figures the first curve from the horizontal zero axis corresponds to $\alpha=2$, the second to $\alpha=0.5$, and the third one to $\alpha=0.2$ (note difference in scale).

(see Appendix E of Ref.18 and references to earlier literature therein).

At low temperature the main contributions into $V_{l}$ for $l \geq 3$ come from $W_{l}$ (Fig.11b). Because the r. h. s. of Eq. (5) has the form of the discrete second derivative and the curve $W_{l}(l)$ is concave, all multiatom interactions are repulsive. On the other hand, in the case under consideration when atoms are supposed to assemble into clusters, there should exist attractive interactions which in our model are necessarily the pair ones [see Eq. (2)].

\section{EXAMPLES FROM METALLIC HETEROEPITAXIAL SYSTEMS}

To illustrate the above formalism with realistic examples of strained epitaxy we consider two metallic heteroepitaxial systems - $\mathrm{Ag} / \mathrm{Pt}$ and $\mathrm{Pt} / \mathrm{Co}$ - which currently are being actively studied both experimentally $19,20,21$ and theoretically22,23. For simplicity we considered 1D case and used the geometry of Ref.2 where the growth on the steps of the closed packed (111) vicinal surface was studied. The position of a deposited atom was relaxed to its equilibrium value in order to find the value $k_{s}$ as the second derivative of the potential near equilibrium. The many-body "potentials" and corresponding parameters were taken 
TABLE I: Parameters corresponding to the Pt/Co heteroepitaxial system

\begin{tabular}{cccc}
$\epsilon$ & $\alpha$ & $k_{p} f^{2}(\mathrm{eV})$ & $V_{N N}(\mathrm{eV})$ \\
\hline $0 \%$ & 0.32 & 0.049 & -0.218 \\
$2 \%$ & 0.25 & 0.100 & -0.179 \\
$3 \%$ & 0.22 & 0.136 & -0.152 \\
\hline
\end{tabular}

from Ref.2 for the Ag/Pt system and from Ref.23 for the Pt/Co system. These potentials were devised specifically to application in the heteroepitaxy and for the (111) surfaces so we expect our results below are quite reliable. Other parameters listed in Table IV were calculated for the atomic pairs relaxed only in vertical position because in our approach we need the first and second derivatives [see discussion after Eq. (2)] calculated in the center of of symmetry of the $1 \mathrm{D}$ cell. In both calculations only the NN interactions were taken into account because the NNN ones were found to be negligeable.

According to our calculations the $\mathrm{Ag} / \mathrm{Pt}$ system has the following parameters: (the energy unit is eV, the length unit $\AA$ ): $V_{\mathrm{NN}}^{p} \approx-0.57, V_{\mathrm{NNN}}^{p} \approx-8.8 \cdot 10^{-3}, k_{p} f^{2} \approx 0.72$, and $\alpha \approx 3.7$. The large value of $\alpha$ means that the relaxation of the strain is very weak (see Fig. 1) so there is no size calibration with the above parameters. The crucial parameter $\alpha$, however, can strongly vary in different systems. For example, according to our estimates based on the potentials of Ref 23 , in $\mathrm{Pt} / \mathrm{Co} \alpha \simeq 0.32$ is an order of magnitude smaller. Because of this the system is quite close to the self-assembly but the misfit strain is still too small. To enhance the misfit, in our model calculations we assumed that the Co underlayer is further compressed (e. g., by means of deposition on an appropriate substrate) by the factor $1-\epsilon$. (see Table $\amalg$ and Fig. (2). But for $\epsilon$ below the critical value $\epsilon_{c} \approx 2 \%$ there is no self-assembly

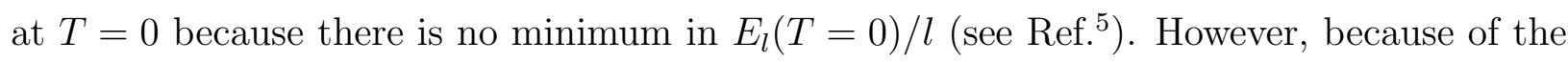
$T$-dependent entropic contribution in the effective energy (3), slightly below $\epsilon_{c}$ the system does exhibit the self-assembly at intermediate temperatures which, however, disappears as $T \rightarrow 0$ (see Fig. 2). Thus, our model predicts a new phenomenon which may be called the entropy driven transient self-assembly. For higher values of strain the $E_{l}(T=0) / l$ curve does have a minimum (see Fig. 2) in which case the qualitative analysis of Ref 


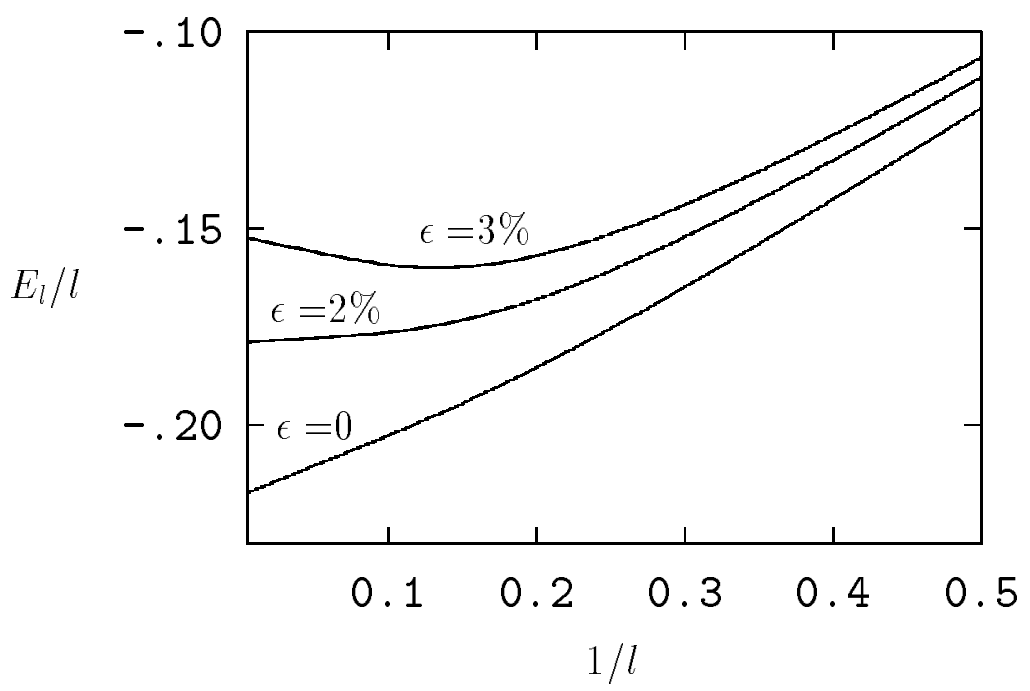

FIG. 2: Length dependence of the reduced energy at zero temperature of the Pt monatomic chains for different values of compression $\epsilon$ of the Co substrate.

\section{CONCLUSION}

In this paper we considered a simple model of strained 1D epitaxy and have shown that similarly to the 2D case the mechanism of self-assembly and size calibration proposed in Ref. $\mathrm{E}$ is operative also in this case. We considered only two explicit examples for which there exist in literature reliable interatomic potentials and in one system already found the size calibration for stressed substrate. In our opinion, this shows that the above phenomena should be as common in 1D as they are in 2D heteroepitaxy. Further argument in favor of this conclusion is that for the size calibration to be plausible the crucial parameter $\alpha=k_{s} / k_{p}$ should be as small as possible. This favors small values of $k_{s}$. However, the geometry considered in the present paper is not quite favorable because of the high coordination of the atoms deposited at the steps with $5 \mathrm{NN}$ atoms of the substrate (see Ref.24) which enhances $k_{s}$. It may be hoped that with lower coordination of the deposited atoms (as in the case of $1 \mathrm{D}$ structures of Ref $\mathrm{d}$ ) the conditions for the size calibration will be more favorable. 


\section{Acknowledgments}

One of us (VT) expresses his gratitude to University Louis Pasteur de Strasbourg and IPCMS for their hospitality.

\section{APPENDIX A}

According to Eq. (7)

$$
D_{l}=\left(\begin{array}{ccccccc}
1+\alpha & -1 & 0 & \ldots & 0 & 0 & 0 \\
-1 & 2+\alpha & -1 & 0 & \ldots & 0 & 0 \\
0 & -1 & 2+\alpha & -1 & 0 & \ldots & 0 \\
\vdots & \vdots & \ddots & \ddots & \ddots & \vdots & \vdots \\
0 & \ldots & 0 & -1 & 2+\alpha & -1 & 0 \\
0 & 0 & \ldots & 0 & -1 & 2+\alpha & -1 \\
0 & 0 & 0 & \ldots & 0 & -1 & 1+\alpha
\end{array}\right),
$$

where $\alpha=k_{s} / k_{p}$. Because matrices $D_{l}$ are tri-diagonal, their determinants satisfy recurrence relations which can be used to calculate all quantities entering Eqs. (4) and (5). The diagonal element of the matrix $G_{l}=1 / D_{l}$ is

$$
G_{l}^{(0)}=r_{l-1} / \operatorname{det} D_{l}
$$

where $r_{l-1}$ is the determinant of the matrix obtained from $D_{l}$ by crossing out its first row and the first column:

$$
r_{l-1}=\left|\begin{array}{cccccc}
2+\alpha & -1 & 0 & \ldots & 0 & 0 \\
-1 & 2+\alpha & -1 & 0 & \ldots & 0 \\
0 & -1 & 2+\alpha & -1 & 0 & 0 \\
\vdots & \vdots & \ddots & \ddots & \ddots & \vdots \\
0 & \ldots & 0 & -1 & 2+\alpha & -1 \\
0 & 0 & \ldots & 0 & -1 & 1+\alpha
\end{array}\right| .
$$


Expanding det $D_{l}$ with respect to the first row we get

$$
\operatorname{det} D_{l}=(1+\alpha) r_{l-1}-r_{l-2}
$$

Comparing this with Eq. (A2) we get

$$
G_{l}^{(0)}=1 /\left(1+\alpha-d_{l-1}\right)
$$

where $d_{l-1}=r_{l-2} / r_{l-1}$. Now, expanding determinant Eq. (A3) with respect to the elements of the first row we get the following three term recurrence relation

$$
r_{l-1}=(2+\alpha) r_{l-2}-r_{l-3} .
$$

But because Eq. (A2) includes only the ratio $r_{l-2} / r_{l-1}$ by dividing Eq. (A6) by $r_{l-2}$ we can transform it to a simpler form

$$
\frac{1}{d_{l-1}}=2+\alpha-d_{l-2} .
$$

The off-diagonal matrix element $G_{l}^{(l-1)}$ of the inverse matrix $G_{l}$ is equal to the ratio of the determinant of the matrix obtained from $D_{l}$ by crossing out its first row and the last column multiplied by $(-1)^{l+1}$ and divided by $\operatorname{det} D_{l}$. As is easy to see from Eq. (A1) the matrix thus obtained is a triangular matrix with the diagonal elements being all equal to -1. Hence its determinant is equal to $(-1)^{l-1}$ and

$$
G_{l}^{(l-1)}=1 / \operatorname{det} D_{l}=\operatorname{det} G_{l} .
$$

From Eq. (A4) we get

$$
G_{l}^{(l-1)}=\frac{1}{\operatorname{det} D_{l}}=\frac{1}{r_{l-1}\left(1+\alpha-d_{l-1}\right)}=\frac{b_{l-1}}{1+\alpha-d_{l-1}}=b_{l-1} G_{l}^{(0)},
$$

where $b_{l-1}=1 / r_{l-1}=d_{l-1} b_{l-2}$ (see the definition of $d_{l-1}$ above). Finally, making the shift $l-1 \rightarrow l+1$ the above formulas can be summarized as

$$
\left\{\begin{array}{l}
G_{l+1}^{(0)}=1 /\left(1+\alpha-d_{l}\right) \\
G_{l+1}^{(l)}=\operatorname{det} G_{l+1}=G_{l+1}^{(0)} b_{l},
\end{array}\right.
$$

where $d_{l}$ and $b_{l}$ are generated by the recursion relations

$$
d_{l+1}=1 /\left(2+\alpha-d_{l}\right) \quad \text { and } \quad b_{l+1}=d_{l+1} b_{l}
$$


initialized by $d_{0}=b_{0}=1$.

1 D. J. Eaglesham and M. Cerullo, Phys. Rev. Lett. 64, 1943 (1990); Y.-W. Mo, D. E. Savage, B. S. Swartzentruber, and M. G. Lagally, Phys. Rev. Lett. 65, 1020 (1990); S. Guha, A. Madhukar, and K. C. Rajkumar, Appl. Phys. Lett. 57, 2110 (1990).

2 R. Notzel, J. Temmyo, and T. Tamamura, Nature (London) 369, 131 (1994); D. Leonard et al., Appl. Phys. Lett. 63, 3203 (1993).

3 A. O. Orlov, I. Amlani, C. S. Lent, and G. L. Snider, Science 277, 928 (1997).

4 J. H. van der Merwe, D. L. Tönsing, and P. M. Stoop, Surf. Sci. 312, 387 (1994); M. Henzler, Surf. Sci. 357-358, 809 (1996); S. Tan, A. Ghazali, and J. C. S. Lévy, Surf. Sci. 369, 360 (1996).

5 C. Priester and M. Lannoo, Phys. Rev. Lett. 75, 93 (1995).

6 K. E. Khor and S. D. Sarma, Phys. Rev. B 62, 16657 (2000).

7 P. Segovia, D. Purdie, M. Hensenberger, and Y. Baer, Nature (London) 402, 504 (1999).

8 J. Dorantes-Dávila and G. M. Pastor, Phys. Rev. Lett. 81, 208 (1998).

9 J. A. Snyman and J. H. van der Merwe, Surf. Sci. 45, 619 (1974).

10 E. Korutcheva, A. M. Turiel, and I. Markov, Phys. Rev. B 61, 16890 (2000).

11 S. C. Erwin, A. A. Baski, L. J. Whitman, and R. E. Rudd, Phys. Rev. Lett. 83, 1818 (1999).

12 C. Uebing and R. Homer, J. Chem. Phys. 95, 7626 (1991).

13 B. G. Orr, D. Kessler, C. Snyder, and L. Sander, Europhys. Lett. 19, 33 (1992).

14 C. Ratsch and A. Zangwill, Surf. Sci. 293, 123 (1993).

15 J. A. Venables, Phys. Rev. B 36, 4153 (1987).

16 J. P. K. Doye and F. Calvo, Phys. Rev. Lett. 86, 3570 (2001).

17 V. I. Tokar and H. Dreyssé, Molec. Phys. 100, 3151 (2002).

18 A. van de Walle and G. Ceder, Rev. Mod. Phys 74, 11 (2002).

19 A. F. Becker, G. Rosenfeld, B. Poelsema, and G. Comsa, Phys. Rev. Lett. 70, 477 (1993).

20 P. Gambardella, M. Blanc, H. Brune, K. Kuhnke, and K. Kern, Phys. Rev. B 61, 2254 (2000).

21 P. Gambardella, M. Blanc, L. Bürgi, K. Kuhnke, and K. Kern, Surf. Sci. 449, 93 (2000).

22 C. Goyhenex and G. Tréglia, Surf. Sci. 446, 272 (2000).

23 C. Goyhenex, H. Bulou, J.-P. Deville, and G. Tréglia, Phys. Rev. B 60, 2781 (1999). 
24 F. Picaud, C. Ramseyer, C. Girardet, and P. Jensen, Phys. Rev. B 61, 16154 (2000). 